\title{
Wahlbeteiligung ist nicht alles! Eine Erwiderung auf Michael Jankowski u.a. in Heft 2/2013 der ZParl
}

\author{
Patrick Horst
}

In Heft 2/2013 der ZParl argumentieren Michael Jankowski, Cord Jakobeit, Philipp Hiller und Nils Thomsen, dass das neue Wahlrecht in Hamburg - ein Zehnstimmenwahlrecht, das die Möglichkeit des Kumulierens und Panaschierens sowohl auf Wahlkreis- als auch auf Landesebene vorsieht - nicht beziehungsweise nur marginal zum Rückgang der Wahlbeteiligung bei der Bürgerschaftswahl 2011 beigetragen habe. ${ }^{1}$ Von stärkerer Bedeutung seien wahlspezifische Faktoren wie der langweilige Wahlkampf, das schwache Kandidatenangebot und die Vorhersehbarkeit des Wahlergebnisses gewesen. In diesem Zusammenhang nehmen sie unter anderem Bezug auf meinen Artikel zum neuen Hamburger Wahlrecht. ${ }^{2}$ Darin wie auch in meiner im selben Heft der ZParl veröffentlichten Analyse zur Hamburger Bürgerschaftswahl $2011^{3}$ bewerte ich das neue Wahlrecht nur als einen Ursachenfaktor für die zurückgehende Wahlbeteiligung „neben anderen Faktoren wie dem weitgehend feststehenden Regierungswechsel, der Inhaltsarmut des Wahlkampfs und der Enthaltung enttäuschter CDU-Wähler“4. Von monokausaler Betrachtung, wie sie mir Jankowski u.a. vorwerfen, kann also keine Rede sein. Richtig jedoch ist, dass ich den demokratiepolitischen Folgen des Wahlrechts und darunter auch der Wahlbeteiligung eine besondere Aufmerksamkeit widme, weil das Wahlrecht vom Gesetzgeber unmittelbar beeinflusst und verändert werden kann. Außerdem wirkt das Wahlrecht seinerseits in vielfältiger, erwünschter und unerwünschter Form auf das Verhalten von Politikern und Wählern ein. Gerade weil es eine solch fundamentale institutionelle Stellschraube im politischen Prozess ist, sollte sich die Politikwissenschaft gefordert sehen, anhand einschlägiger Kriterien und wohlbegründeter Maßstäbe die Geeignetheit bestimmter Wahlrechtsentwürfe zu beurteilen.

Zur Beurteilung eines Wahlrechts und der Ableitung von politischen Handlungsempfehlungen reicht es keineswegs aus, sich allein auf die Wahlbeteiligung als Kriterium zu beschränken, wie es Jankowski u.a. tun. Sicherlich ist die Wahlbeteiligung in der Demokratie ein hohes Gut, aber die Autoren weisen selbst darauf hin, dass viele kontingente Ursachenfaktoren in sie einfließen. Deshalb ist die Wahlbeteiligung nur ein Kriterium neben anderen - und beileibe nicht das wichtigste ${ }^{5}$-, um ein Wahlsystem zu bewerten. Wenn im Hamburger Fall darauf besonderes Gewicht gelegt wurde, so hing dies auch damit zusammen, dass es das erklärte Ziel der Wahlrechtsinitiatoren von „Mehr Demokratie“ war, mit dem neuen Wahlrecht die als niedrig empfundene Wahlbeteiligung wieder zu steigern. Wenn ein Wahlsystem

1 Vgl. Michael Jankowski / Cord Jakobeit / Philipp Hiller / Nils Thomsen, Mehr Wahl, mehr Qual? Zum Zusammenhang von Wahlbeteiligung und neuem Wahlrecht in Hamburg, in: ZParl, 44. Jg. (2013), H. 2, S. $264-275$.

2 Vgl. Patrick Horst, Das neue Hamburger Wahlrecht auf dem Prüfstand: kontraproduktiv, aber schwer reformierbar, in: ZParl, 42. Jg. (2011), H. 4, S. 707 - 724, insbesondere S. 713 f. und S. 721 ff.

3 Vgl. ders., Die Wahl zur Hamburger Bürgerschaft vom 20. Februar 2011: Ehemalige „HamburgPartei“ erobert absolute Mehrheit zurück, in: ZParl, 42. Jg. (2011), H. 4, S. 724 - 744.

4 Wörtliches Zitat ebenda, S. 733.

5 So aber Michael Jankowski / Cord Jakobeit / Philipp Hiller / Nils Thomsen, a.a.O. (Fn. 1), S. 264, Fußnote 3. 
aber das Gegenteil von dem erreicht, was es beabsichtigt, dann sollte dies schon ein Grund zum Nachdenken sein. Auf jeden Fall rechtfertigt es die Frage, ob dem Wahlrechtskonzept möglicherweise ein gedanklicher Fehler zugrunde lag oder es von falschen Voraussetzungen ausging.

Die moderne Wahlsystemforschung ist längst über das Stadium hinweg, in dem Befürworter des Verhältnis- oder des Mehrheitswahlrechts ihr Plädoyer einseitig an dem Ziel einer spiegelbildlichen Repräsentation gesellschaftlicher Kräfte oder handlungsfähiger Regierungen ausrichten konnten. Heutzutage ist es üblich, Wahlsysteme an einem Bündel von Kriterien zu messen, die möglichst alle mehr oder weniger zufriedenstellend erfüllt werden sollten. Dieter Nohlen nennt zum Beispiel in seinem einschlägigen Werk fünf solcher Kriterien: Repräsentation, Konzentration, Partizipation, Einfachheit und Legitimität. ${ }^{6}$ Bei Jankowski u.a. dreht sich alles um ein einziges dieser fünf Kriterien: die Partizipation. Dabei ist es erst einmal richtig, dass die Abkehr vom starren Listenwahlrecht und die eingeräumten Möglichkeiten zum Kumulieren und Panaschieren die Gestaltungsmöglichkeiten der Wähler steigern, wie die Autoren feststellen. Ob damit aber auch „eine bessere Berücksichtigung des Wählerwillens“7 einhergeht, ist fraglich. Das setzt zum einen voraus, dass der Wähler weiß, wie seine Stimmen wirken - und sich auch über mögliche nichtintendierte Folgen seiner Stimmabgabe im Klaren ist. Daran sind erhebliche Zweifel angebracht, die auch nicht dadurch gemindert werden, dass eine knappe Mehrheit der Hamburger Wähler das Wahlrecht als nicht zu kompliziert bezeichnete. Nach eingehender Beschäftigung mit der komplexen Materie des Hamburger Wahlrechts erscheint mir gesichert, dass viele dieser Wähler ihre kognitiven Fähigkeiten - sei es aus Gründen individueller oder sozialer Erwünschtheit - zu optimistisch einschätzen. Zum anderen ist es prinzipiell diskussionswürdig, ob die vielfach zersplitterte Willensbekundung des Wählers dem gewählten Repräsentanten eine bessere Ermittlung und Umsetzung dieses Willens ermöglicht als eine einheitliche Stimmabgabe zugunsten einer Partei oder eines Kandidaten.

Nimmt man trotz dieser Zweifel und trotz der gesunkenen Wahlbeteiligung (insbesondere der sozial schwachen Bevölkerung) an, dass das neue Wahlrecht in Hamburg zur Steigerung des Partizipationsziels beizutragen vermag, so müsste für seine positive Bewertung immer noch gezeigt werden, dass es auch den anderen Kriterien eines guten Wahlrechts gerecht wird. In dieser Hinsicht seien meine erheblichen Zweifel wiederholt: In Bezug auf das Repräsentationsziel konnten einzig bei der deskriptiven Repräsentation (von Minderheiten, Frauen und Jüngeren) leichte Verbesserungen festgestellt werden, die aber kaum ursächlich auf das neue Wahlrecht zurückzuführen waren. Hinsichtlich der - wichtigeren - substantiellen Repräsentation und der Responsivität der Abgeordneten gegenüber dem Wähler, Nohlen würde dies unter das Kriterium der Konzentration fassen, bewirkte das neue Wahlrecht demgegenüber bisher nichts Positives. Die Arbeitsfähigkeit des Parlaments und der Fraktionen wurde durch die extrem freie, von den Parteien nur noch schwer zu steuernde Kandidatenauswahl stark beeinträchtigt. Das Übergewicht des Senats und des Ersten Bürgermeisters gegenüber der Bürgerschaft ist weiter gewachsen. Dazu trug nicht zuletzt die mit dem Wahlrecht weiter verschärfte Personalisierung des Wahlkampfes bei. Diese beschränkte sich fast ausschließlich auf die Spitzenkandidaten, erfasste aber in der Regel nicht

6 Vgl. Dieter Nohlen, Wahlrecht und Parteiensystem. Zur Theorie und Empirie der Wahlsysteme, Opladen 2009, S. 166 - 173.

7 Michael Jankowski / Cord Jakobeit / Philipp Hiller / Nils Thomsen, a.a.O. (Fn. 1), S. 265 f. 
den Kandidatenwettbewerb in den Wahlkreisen. Dafür war zwar nicht in erster Linie das neue Wahlrecht verantwortlich, sondern die Parteien und Kandidaten selbst, weil sie den innerparteilichen Wettbewerb dämpften. Trotzdem: Ein Wahlrecht hat nicht nur den Wählern, sondern auch den spezifischen Anforderungen eines konkreten politischen Systems und der in ihm agierenden Parteien zu dienen. Ausgerechnet von Hamburgs Feierabendparlamentariern eine Belebung des Wahlkampfes zu erwarten, war eine bemerkenswerte Fehlkalkulation der Wahlrechtsinitiatoren. Dass darüber hinaus das neue Wahlrecht das Kriterium der Einfachheit in nahezu sträflicher Weise vernachlässigt, bestreiten auch Jankowski u.a. nicht. Allerdings übersehen sie, dass sich dies - unbeschadet von Meinungsumfragen, die das neue Wahlrecht mehrheitlich besser bewerten als das alte ${ }^{8}$ - über kurz oder lang auch abträglich auf die Legitimität des Wahlsystems und der parlamentarischen Demokratie insgesamt auswirken könnte. Die mittelbaren, quantitativ gar nicht zu erfassenden Entpolitisierungswirkungen des Wahlrechts sind viel schwerwiegender als der unmittelbare Rückgang der Wahlbeteiligung.

Erstaunlich ist, dass Jankowski u.a. ihre Argumentation ausschließlich auf die von ihnen durchgeführte Wahltagsumfrage unter Wählern und Nichtwählern stützen, mir dabei vorwerfen, diese „einseitig“ zu interpretieren und meine Schlussfolgerung des Zusammenhangs zwischen Wahlrecht und sinkender Wahlbeteiligung ,weitestgehend ohne eine empirische Grundlage“ zu ziehen. ${ }^{9}$ Dieser Vorwurf lässt sich trefflich umkehren: Während sich meine Schlussfolgerungen gerade nicht allein auf uninterpretiert hingenommene Meinungsumfragen stützen, sondern auf ein theoretisches Vorverständnis der Wahlsystem- und Parlamentarismusforschung sowie vor allem auf eine Realanalyse des tatsächlichen Wahlverhaltens, versäumen es Jankowski u.a., ihre Wahlanalyse ${ }^{10}$ mit der Bewertung des neuen Wahlrechts interpretierend zu verknüpfen. Dies hätte sie vor manchen einseitigen Fehlinterpretationen bewahrt und davor, die Antworten der Befragten durchgängig für bare Münze zu nehmen. Denn welche Erkenntnis lässt sich zum Beispiel aus der Tatsache gewinnen, dass drei Viertel der Hamburger Nichtwähler sich vom Wahlkampf nicht angesprochen fühlten, zwei Drittel von den Kandidaten nicht überzeugt waren und gut die Hälfte sich für mehr Volksentscheide aussprach? ${ }^{11}$ Mit Fug und Recht darf angenommen werden, dass dies bei früheren Wahlen auch schon der Fall war. Das Wahlrecht war unter den vorgegebenen Antwortmöglichkeiten - sieht man einmal von dem scheinbar sicheren Wahlausgang ab, der zumindest 2008 nicht in dem Maße gegeben war - der einzige Faktor, der sich gegenüber 2008 beziehungs-

8 Siehe dazu ebenda, S. 272, Tabelle 2.

9 Ebenda, S. 267.

10 Die dem Artikel von Michael Jankowski u.a. zugrunde liegende, im Auftrag des Verfassungsausschusses der Hamburgischen Bürgerschaft erstellte Evaluationsstudie enthält auf den Seiten 40 bis 84 durchaus eine Wahlanalyse, die auf den Daten des Statistikamtes Nord beruht. Bemerkenswerterweise werden aus den Befunden (erhebliche Durcheinanderwürfelung der Listen, niedrige Wahlbeteiligung in den sozial schwachen Wohngebieten, überproportional hoher Anteil ungültiger Stimmen vor allem unter älteren Wählern) keine Schlussfolgerungen für die Bewertung des Wahlrechts gezogen. Vgl. Cord Jakobeit / Philipp Hiller / Nils Thomsen / Michael Jankowski / Max Pritsch / Daniel Jäckel, Evaluation des neuen Hamburger Wahlrechts bei der Bürgerschaftswahl 2011. Ergebnisse einer Wähler- und Nichtwählerbefragung, Hamburg, 30. Juni 2011, in: Bürgerschaft der Freien und Hansestadt Hamburg, Drucksache 20/1024 vom 7. Juli 2011.

11 Vgl. Michael Jankowski / Cord Jakobeit / Philipp Hiller / Nils Thomsen, a.a.O. (Fn. 1), S. 274, Tabelle 5 . 
weise $2004^{12}$ wirklich fundamental geändert hatte. Insofern kann mit einiger Sicherheit vermutet werden, dass der ungewöhnlich starke Rückgang der Wahlbeteiligung seit 2004 vor allem mit dem neuen Wahlrecht zu tun hatte. Zuvor war die Wahlbeteiligung in Hamburg nicht gesunken, sondern zwischen 1991 und 2001 sogar gestiegen. ${ }^{13}$

Im Übrigen ergibt schon eine simple überschlägige Rechnung, dass das neue Wahlrecht - die Antworten der Nichtwähler beim Wort genommen und die Repräsentativität der Stichprobe angenommen - für mindestens die Hälfte des gemessenen Rückgangs der Wahlbeteiligung von 6,2 Prozentpunkten verantwortlich war: Laut Befragung der Autoren gaben 7,3 Prozent der Nichtwähler an, dass das neue Wahlrecht für sie „der ausschlaggebende Grund“ für ihre Wahlenthaltung gewesen sei. ${ }^{14}$ Bei über 40 Prozent Nichtwählern (die Wahlbeteiligung betrug 2011 in Hamburg 57,3 Prozent) macht diese Gruppe allein gut drei Prozent der Wahlberechtigten aus. Und hier sind die 20 Prozent der Nichtwähler, für die das neue Wahlrecht einen „wichtigen“ oder einen „weniger wichtigen Grund unter vielen“ für die Nichtwahl darstellte, noch nicht eingerechnet. ${ }^{15}$

Meine Prognose, dass das neue Wahlrecht eine „Reformbaustelle“ bleiben werde, hatte ich relativiert: „sofern die Parteien aus Angst vor dem Wähler davor nicht zurückschrecken“16. Dass Jankowski u.a. diese Relativierung unterschlagen, ist unschön. Schwerer wiegt, dass genau dieser Fall, der sich im Sommer 2011 schon abzeichnete, nun eingetreten ist - und die Autoren der Evaluationsstudie sich vorhalten lassen müssen, dass sie mit ihrer schönfärberischen Bewertung des neuen Wahlrechts dazu beigetragen haben. Das Ausbleiben einer erneuten Wahlrechtsreform in Hamburg erfolgte nämlich keinesfalls - jedenfalls nicht bei den großen Parteien SPD und CDU sowie bei Teilen der Grünen - aus Überzeugung, sondern allein aus dem Kalkül heraus, es sich nicht wie die CDU nach 2004 mit dem Wähler und der Interessengruppe „Mehr Demokratie“ zu verscherzen. ${ }^{17}$ Eine neuerliche Reform mittlerer Größenordnung hätte deshalb nur im Konsens der Parteien verabschiedet werden können - und hätte darüber hinaus eindeutige, wissenschaftlich begründete Handlungsempfehlungen der Wahlrechtsevaluatoren verlangt. Diese sind bis heute ausgeblieben. ${ }^{18}$

12 Schon 2008 wurde nach einem personalisierten Verhältniswahlrecht mit sechs Stimmen (eine auf Landes-, fünf auf Wahlkreisebene) gewählt, das jedoch noch nicht so weitreichende Möglichkeiten zum Kumulieren und Panaschieren einräumte.

13 Vgl. hierzu die anschauliche Graphik bei Michael Jankowski / Cord Jakobeit / Philipp Hiller / Nils Thomsen, a.a.O. (Fn. 1), S. 266.

14 Vgl. Cord Jakobeit / Philipp Hiller / Nils Thomsen / Michael Jankowski / Max Pritsch / Daniel Jäckel, a.a.O. (Fn. 10), S. 133, Abbildung 8.6.

15 Ebenda.

16 Vgl. Patrick Horst, a.a.O. (Fn. 2), S. 723.

17 Dies lässt sich dem Wortprotokoll der öffentlichen Sitzung des Verfassungsausschusses vom 13. September 2011, Nr. 20/05, S. 21 - 51, sowie dem abschließenden Bericht des Verfassungsausschusses vom 1. Juli 2013, Drucksache 20/8562 entnehmen.

18 Die zwei kleineren Reformempfehlungen, die Jankowski u.a. nun verspätet nachreichen, entsprechen genau dem, was ich schon 2011, nur detaillierter, als „kleine Lösung“ vorgeschlagen habe. Leider versäumen sie es, auf diese Tatsache hinzuweisen. Vgl. Michael Jankowski / Cord Jakobeit I Philipp Hiller / Nils Thomsen, a.a.O. (Fn. 1), S. 275 mit Patrick Horst, a.a.O. (Fn. 2), S. 723. 